\title{
Effect of Quinoa Seeds (Chenopodium quinoa) in Diet on some Biochemical Parameters and Essential Elements in Blood of High Fructose-Fed Rats
}

\author{
Paweł Paśko • Paweł Zagrodzki • Henryk Bartoń • \\ Joanna Chłopicka $\cdot$ Shela Gorinstein
}

Published online: 23 November 2010

(C) The Author(s) 2010. This article is published with open access at Springerlink.com

\begin{abstract}
The effect of Chenopodium quinoa seeds on lipid profile, glucose level, protein metabolism and selected essential elements $(\mathrm{Na}, \mathrm{K}, \mathrm{Ca}, \mathrm{Mg}$ ) level was determined in high - fructose fed male Wistar rats. Fructose decreased significantly LDL $[42 \%, p<0.01]$ and activity of alkaline phosphatase $[20 \%, p<0.05]$, and increased triglycerides level $[86 \%, p<0.01]$. The analysis of blood of rats fed quinoa indicated, that these seeds effectively reduced serum total cholesterol [26\%, $p<0.05]$, LDL [57\%, $p<0.008]$ and triglycerides $[11 \%, p<0.05]$ when compared to the control group. Quinoa seeds also significantly reduced the level of glucose $[10 \%, p<0.01]$ and plasma total protein level $[16 \%$, $p<0.001]$. Fructose significantly decreased HDL $[15 \%, p<$ 0.05 ] level in control group but when the quinoa seeds were added into the diet the decrease of HDL level was inhibited. Quinoa seeds did not prevent any adverse effect of increasing triglyceride level caused by fructose. It was shown in this study that quinoa seeds can reduce most of the adverse effects exerted by fructose on lipid profile and glucose level.
\end{abstract}

\footnotetext{
P. Paśko $(\bowtie) \cdot$ P. Zagrodzki $\cdot$ H. Bartoń $\cdot$ J. Chłopicka Department of Food Chemistry and Nutrition, Medical College, The Jagiellonian University,

Medyczna 9,

30-688, Kraków, Poland

e-mail: paskopaw@poczta.fm

P. Zagrodzki

Department of Nuclear Physical Chemistry, Henryk

Niewodniczański Institute of Nuclear Physic,

Kraków, Poland

S. Gorinstein

Institute for Drug Research, School of Pharmacy, The Hebrew University-Hadassah Medical School,

Jerusalem, Israel
}

Keywords Biochemical parameters $\cdot$ Fructose $\cdot$ Quinoa seeds $\cdot$ Rats $\cdot$ Essential element

\section{Introduction}

There is considerable evidence that administration of fodders enriched with fructose to rats induced adverse metabolic alterations and oxidative stress leading to hypertriglyceridemia, high blood pressure, obesity and impaired glucose tolerance and insulin resistance [1-3]. It was also demonstrated that in rats fed with high-fructose diet, the free radicals concentration was three times higher than in the control group [4]. Quinoa seeds (Chenopodium quinoa) contain significant amounts of phytochemicals including: flavonoids, phenolic acids, squalene, phytosterol, saponins, fat-soluble vitamins, fatty acids, trace elements and other compounds which can influence biochemical parameters in organisms [5-8]. Until now, a few reports have dealt with the influence of quinoa seeds on lipid metabolism and hypocholesterolemia [9]. However, it is well-known that some other pseudocereal plants such as amaranth or buckwheat exert lowering effect on total serum cholesterol [10-15].

The present study was designed to assess the impact of fructose addition on the biochemical parameters of blood of rats fed diet with quinoa seeds. The experimental model with adding $31 \%$ of fructose to diet was applied purposefully to induce oxidation. The following plasma parameters were taken into consideration: lipid profile - total cholesterol, high density lipoproteins (HDL), low density lipoproteins (LDL), triglycerides, glucose, urea, uric acid, creatinine, albumins, total protein concentrations, and alkaline phosphatase activity. Concentrations of sodium, potassium, magnesium, calcium and chloride were also determined. 


\section{Material and Methods}

\section{Plant Material}

Quinoa seeds (Chenopodium quinoa Willd.) where cropped in Bolivia in 2006 and imported by Biofuturo Co., Kraków, Poland. Voucher specimen was deposited in the Department of Food Chemistry and Nutrition, Medical College Jagiellonian University (No \# CQ/PP/PL 1021).

The dry seeds were packaged in moisture-proof containers, and stored in a freezer. They were conditioned at room temperature before use. The detailed composition of the seeds was not studied in this work.

\section{Preparation of Diets}

Diets were formulated according to the following scheme: compounds in constant amounts (i.e. their amounts were the same in each diet) [g/kg fodder]: casein 200, rapeseed oil 50, chalk 28, calcium monophosphate 29, lecithin 10, sodium chloride 3 , cellulose 50, mixture of vitamins and microelements 10 (Premix LPM, BASF, Poland). Two groups ( $\mathrm{C}$ - control; $\mathrm{CF}$ - control with fructose) were given either standard fodder, which contained $620 \mathrm{~g} / \mathrm{kg}$ corn starch or a fodder in which $310 \mathrm{~g} / \mathrm{kg}$ of corn starch was substituted with $310 \mathrm{~g} / \mathrm{kg}$ of fructose, respectively. In the fodder for the group "Q" $310 \mathrm{~g} / \mathrm{kg}$ of corn starch was substituted with $310 \mathrm{~g} / \mathrm{kg}$ of quinoa seeds, and in the fodder for the group "QF" next $310 \mathrm{~g} / \mathrm{kg}$ of corn starch was substituted with $310 \mathrm{~g} / \mathrm{kg}$ of fructose. The energy density, total amount of proteins, fat, and carbohydrates in the diets is presented in Table 1.

\section{Animals}

Twenty-four male Wistar rats (mean weight $245.4 \pm 7.0 \mathrm{~g}$ ) were purchased from the Animal House of Jagiellonian University. Each tested group: control group (C); control group with $31 \%$ of fructose (CF); group fed diet with quinoa seeds (Q); group fed diet with quinoa seeds and with $31 \%$ of fructose (QF), consisted of six animals. The

Table 1 The energy density, total amount of proteins, fat, and carbohydrates in the diets $(\mathrm{C}$ - control group; $\mathrm{CF}$ - control group with $31 \%$ of fructose; Q - group fed diet with quinoa seeds; QF - group fed diet with quinoa seeds and with $31 \%$ of fructose)

\begin{tabular}{lrrrr}
\hline & C & CF & Q & \multicolumn{1}{c}{ QF } \\
\hline Energy [kcal/g fodder] & 3.26 & 3.32 & 3.27 & 3.33 \\
Protein [g/kg fodder] & 142.4 & 141.1 & 183.9 & 182.7 \\
Fat [g/kg fodder] & 52.8 & 51.0 & 73.4 & 71.6 \\
Carbohydrates [g/kg fodder] & 573.2 & 592.4 & 483.9 & 503.1 \\
\hline
\end{tabular}

rats were housed in metal-plastic cages (three animals per cage) and kept in an air-conditioned animal-room at a temperature of $22 \pm 2{ }^{\circ} \mathrm{C}$, with a relative humidity of $50 \pm$ $5 \%$. The animals' room was on a $12 \mathrm{~h}$ daily lighting-period cycle, and the rats were kept there for the whole period of investigation, i.e. five weeks. The rats had unlimited access to fodder and tap water. The protocols for animal experiments were approved by the Animal Experimentation Committee of Jagiellonian University.

\section{Sample Collections}

Blood samples were taken from aorta under general anaesthesia following intraperitoneal thiopental injection.

\section{Analytical Procedure}

Rats' plasma biochemical parameters were determined by using the automatic analyzer country of Konelab 30.

\section{Statistical Procedure}

Values are given as mean \pm standard deviation (SD). The statistical analyses of biochemical parameters were conducted using the Statistica 6.1 PL software (StatSoft, Inc.). A type of distribution of analyzed variables was determined by chi-square test. A variance homogeneity was analyzed using the Hartley test. In order to compare mean values a one-way ANOVA test was applied. Kruskal-Wallis test, followed by Dunn's post hoc test, was applied for statistical evaluations of parameters with either non-Gaussian distribution or non-homogenous variance. The critical significance level was set as $p<0.05$.

\section{Results}

The results of biochemical parameters for every group are gathered in Fig. 1 and Table 2. The most significant changes were observed for lipid profile (Fig. 1).

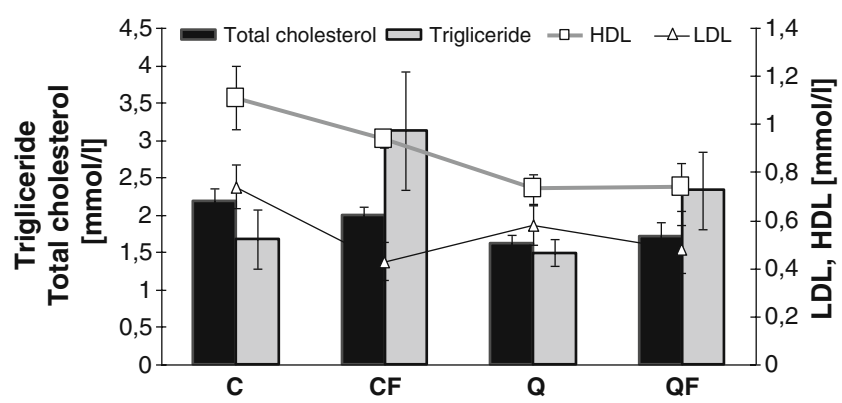

Fig. 1 Effects of fructose and quinoa seeds on lipid profile (total cholesterol, trigliceride, HDL, LDL) in rats' blood*. * Values are given as mean $\pm \mathrm{SD}$ for six animals 
Table 2 Effects of fructose and quinoa seeds on glucose, uric acid, urea, creatinine, total protein, albumin, selected essential elements level and activity of alkaline phosphatase in rats' blood** Values represent means \pm SD for six animals

\begin{tabular}{|c|c|c|c|c|c|}
\hline \multirow[t]{2}{*}{ Parameters } & \multicolumn{4}{|c|}{ Groups of rats } & \multirow[t]{2}{*}{ Significant differences } \\
\hline & $\mathrm{C}$ & $\mathrm{CF}$ & Q & QF & \\
\hline Glucose $[\mathrm{mmol} / 1]$ & $8.9 \pm 0.4$ & $9.42 \pm 0.8$ & $7.97 \pm 0.6$ & $8.4 \pm 0.4$ & C vs. Q $p<0.01$ \\
\hline Uric acid $[\mathrm{mmol} / \mathrm{l}]$ & $24.4 \pm 4.4$ & $33.8 \pm 2.9$ & $26.5 \pm 3.2$ & $34.7 \pm 7.8$ & $\mathrm{CF}$ vs. $\mathrm{C} p<0.001 ; \mathrm{QF}$ vs. Q $p<0.04$ \\
\hline Urea $[\mathrm{mmol} / \mathrm{l}]$ & $10.7 \pm 0.6$ & $10.85 \pm 1.4$ & $9.4 \pm 1.9$ & $8.75 \pm 1.5$ & - \\
\hline Creatinine $[\mathrm{umol} / 1]$ & $32.1 \pm 3.4$ & $29.5 \pm 3.8$ & $32.3 \pm 5$ & $28.6 \pm 6$ & - \\
\hline Total protein $[\mathrm{g} / 1]$ & $62.3 \pm 1.2$ & $62.6 \pm 2.3$ & $52.5 \pm 1.2$ & $51.4 \pm 3.1$ & C vs. $\mathrm{Q} p<0.001$ \\
\hline Albumin $[\mathrm{g} / \mathrm{dl}]$ & $34.4 \pm 0.9$ & $34.2 \pm 1.1$ & $31.3 \pm 0.8$ & $31.3 \pm 2$ & - \\
\hline Alkaline phosphatase [U/1] & $239.5 \pm 26$ & $190.5 \pm 13$ & $228.8 \pm 25$ & $192.8 \pm 14$ & CF vs. C $p<0.05$ \\
\hline \multicolumn{6}{|l|}{ Essential elements } \\
\hline $\mathrm{Na}[\mathrm{mmol} / 1]$ & $139.5 \pm 0.8$ & $140.0 \pm 1.5$ & $138.8 \pm 1.2$ & $136.7 \pm 2.2$ & - \\
\hline $\mathrm{K}[\mathrm{mmol} / 1]$ & $3.48 \pm 0.10$ & $3.53 \pm 0.21$ & $3.6 \pm 0.2$ & $3.53 \pm 0.1$ & - \\
\hline $\mathrm{Mg}[\mathrm{mmol} / \mathrm{l}]$ & $0.77 \pm 0.14$ & $0.77 \pm 0.06$ & $0.74 \pm 0.04$ & $0.74 \pm 0.05$ & - \\
\hline $\mathrm{Ca}[\mathrm{mmol} / \mathrm{l}]$ & $2.50 \pm 0.04$ & $2.47 \pm 0.07$ & $2.6 \pm 0.3$ & $2.38 \pm 0.08$ & - \\
\hline $\mathrm{Cl}[\mathrm{mmol} / \mathrm{l}]$ & $102.6 \pm 1.2$ & $103.3 \pm 1.4$ & $100.9 \pm 0.9$ & $98.2 \pm 2.1$ & - \\
\hline
\end{tabular}

Quinoa seeds significantly decreased the level of total cholesterol in comparison to control group (C vs. Q). High dietary fructose did not influence total cholesterol level in any group when compared $\mathrm{CF}$ vs. C or QF vs. Q.

LDL level was significantly decreased in group fed diet with quinoa seeds as compared with control group ( $\mathrm{C}$ vs. Q; $p<0.008)$. In all fructose-fed groups LDL level fell in comparison to appropriate non-fructose-fed rats, and this difference was statistically significant between groups $\mathrm{C}$ vs. CF $(p<0.01)$.

Fructose significantly decreased the HDL level in control group (C vs. CF; $p<0.05$ ) but when the quinoa seeds were added into the diet, the decrease of HDL level was not observed (Q vs. QF).

The triglycerides level significantly decreased after adding quinoa seeds to the diet (C vs. Q; $p<0.05$ ). In all fructose-fed groups triglycerides concentration increased in comparison to non-fructose-fed group, significant differences were observed at $p<0.01$.

Effects of fructose feeding and quinoa seeds on protein metabolism were diverse (Table 2). Urea concentration was unchanged in control group as a result of fructose addition to the fodder, but decreased insignificantly in group $\mathrm{Q}$ as a result of quinoa addition to the diet.

The dietary fructose brought about increase in uric acid concentration regardless of quinoa adding. Statistically significant differences were noted in control groups (CF vs. C; $p<0.001)$ and between groups $\mathrm{QF}$ and $\mathrm{Q}(p<0.04)$.

Fructose decreased insignificantly the level of creatinine in all groups. Adding quinoa seeds to the diet did not cause significant differences of creatinine level (C vs. Q).

Plasma glucose level (Table 2) rose in fructose-fed groups, in control groups (C vs. $\mathrm{CF}$ ) and quinoa groups
(Q vs. QF), but this increase was not significant. When quinoa-fed groups were compared with control groups it was apparent that quinoa significantly decreased the level of glucose (C vs. Q; $p<0.01)$.

In fructose-fed rats, alkaline phosphatase activity was significantly lower than in the non-fructose groups (Table 2). No influence of fructose on total proteins and albumins concentrations was observed. Quinoa seeds decreased plasma total protein level in rats (C vs. Q; $p<0.001$ ) by $16 \%$.

The level of selected mineral elements $(\mathrm{Na}, \mathrm{K}, \mathrm{Mg}, \mathrm{Ca})$ and chloride (Table 2) did not depend on addition of fructose nor quinoa seeds to the diet.

\section{Discussion}

Until now most of the studies on diets enriched with fructose have focused on effects induced by very high content of dietary fructose. In animal studies fructose was a source of $45-66 \%$ of fodder energetic value, and in human studies the value rose to even $90 \%$ [16].

With regard to reports that long-lasting fructose consumption could cause adaptive changes in healthy animals that in turn masked syndromes of such disturbances as in lipid metabolism [17]; in this study we chose the shorter period for the experiment.

In present study the plasma cholesterol concentration was reduced by consumption of quinoa seeds. This finding is in accordance with previous reports $[10,15,18]$ that some proteins from pseudocereals (amaranth, buckwheat or quinoa) can influence the total cholesterol serum level.

Other authors suggest that the hypocholesterolemic effect of quinoa could be produced by the fiber, saponins 
or squalene, which are also present in these seeds [7-9]. Dietary fiber from these grains can inhibit absorption of dietary cholesterol [9] and can bind to biliary acid, which may increase cholesterol catabolism or fermentation of the fiber in the colon and produce short-chain fatty acids contributing to decrease of cholesterol synthesis in liver [12]. Takao et al. [9] observed suppression in the expression of HMG-CoA reductase by quinoa protein. Squalen is regarded as being responsible for the mechanism of these changes, because it is known as inhibitor of this enzyme. The cholesterol-lowering effects of saponins may be mediated by the decreased intestinal absorption of cholesterol. It was proved that saponins from soya could increased faecal bile acid and neutralize sterol excretion [19].

In the light of studies of Benado et al. [20], who revealed that LDL cholesterol concentration increased with the growing fructose rate in food energetic value, our results concerning the impact of fructose addition on LDL cholesterol level are different. The higher content of dietary fructose caused the decrease of plasma LDL cholesterol level (Fig. 1). In contrast with our results, Lewis et al. [21] also reported that feeding rats a diet containing $60 \%$ of fructose for five weeks caused a large rise in LDL level in comparison to the control group fed a standard diet. The discrepancy with our observations could result from applied nutritional model ( $31 \%$ of fructose) in present study. LDL levels were decreased significantly in groups fed with quinoa seeds as compared with control group (Fig. 1). The LDL lowering properties of quinoa seeds may be due to some proteins as well as unsaturated fatty acids and tocopherols. Similar observation in the LDL level caused by other pseudocereal proteins (amaranth protein concentrate) was reported by Escudero et al. [12] and Plate and Areas [14].

Our results showed that quinoa seeds added to the rats' diet inhibited decreasing in HDL level caused by fructose, but it did not stimulate any increase in HDL concentration (Fig. 1). The results of other authors are conflicting. The opposite one to our observation, i.e. decrease in HDL level as a result of quinoa seeds consumption was made by Takao et al. [9]. In analogous works Escudero et al. [12] and Plate and Areas [14] showed that amaranth seeds increased HDL level in rats fed normal diet. In opposition to our results, Lewis et al. [21] observed higher HDL cholesterol concentration in fructose-fed rats in reference to the control group. Such results were also obtained by Benado et al. [20]. Future works on these aspects are expected to reveal the reasons for discrepancies.

In line with our results, Busserolles et al. [22] found that fructose-rich diet induced hypertriglyceridemia in rats after two weeks of the experiment. There was a two-fold increase in plasma triglycerides concentration compared with the control group. The fructose content in the diet applied in the above study was $34 \%$, quite similar to our model. Lewis et al. [21], in the research on rats fed a diet with high fructose level $(60 \%)$, they also reported an increase in triglycerides concentration in comparison to the control group. The above-mentioned changes could be caused by triglycerides overproduction in hepatocytes as a consequence of fructose overload and therefore increased lipogenesis and overproduction of VLDL.

In our study quinoa seeds did not influence positively the triglyceride level. However, Takao et al. [9] observed improving in level of triglyceride level after adding protein fraction isolated from quinoa seeds. Similarly, Escudero et al. [12] observed a significant decrease in triglyceride level in comparison to control group after adding amaranth seeds to the diet.

It is thought that fructose consumption leads to a significant increase in blood glucose level. We also found the same phenomenon, but the change was non-significant in the most of fructose-fed groups in our experiment. When we compared quinoa fed groups with the control group, we could observe that quinoa seeds significantly decreased the level of glucose. Chemical compounds which were present in quinoa seeds (i.e., tocopherols, polyphenols) $[6,7] \mathrm{might}$ be able to improve glucose level, and this effect was already observed after adding rice bran to the diet [23]. Our results suggest also, that in the presence of fructose in diet, quinoa seeds protect rats' organism against increase of glucose level caused by fructose. It was shown that fructose did not affect blood glucose level [24], or brought about only temporary hyperglycemia [25] or moderate hyperglycemia [26]. Such diversity of results could be caused by differences in the amount of fructose intake and experiments durations.

The increased level of plasma urea and uric acid shown in our experiment after fructose ingestion was described in literature previously [27]. This effect could be explained by the fact that fructose metabolism in kidneys utilizes ATP as the source of phosphates for phosphorylation process and it leads to purine and pyrimidine nucleotides degradation. In a human study, the significant increase of urea and purine bases derivates (xanthine and uric acid) concentrations was demonstrated after intravenous injection of $10 \%$ fructose solution ( $0.7 \mathrm{~g}$ fructose $/ \mathrm{kg}$ body weight) [27]. The increase in concentration of uric acid can be also explained as a rise in body purine catabolism due to fructose action. In consequence, increased uric acid level can enhance antioxidative protection of organism, since this compound is known as one of the strongest water-soluble antioxidants.

The high fructose diet had various impacts on creatinine concentration. Creatinine is produced as a product of phosphocreatine degradation and is excreted into urine. If kidneys are not able to eliminate protein metabolic byproducts, the level of plasma creatinine increases and this parameter has been used to measure the chronic renal failure. We suppose that kidneys of most of the animals in 
the experiment worked properly and this is the reason that there were no significant changes in creatinine level between fructose-fed and fructose-free animals. Similar results were achieved by Kizhner and Werman [28]. Quinoa seeds did not influence creatinine level and the similar observation was presented by Ardiansyah et al. [23] after adding rice bran fractions to the diet.

To our knowledge, there is no relevant reference in the literature regarding decrease in alkaline phosphatase activity observed in our experiment in groups fed high-fructose diet. Protein malnutrition is often associated with increased activity of alkaline phosphatase [29]. Obatolu et al. [29] observed that malted maize and soybean caused significant increase in activity of alkaline phosphatase in comparison with non protein diet. This enzyme in non-specific and the interpretation of the results therefore is ambiguous.

Total protein levels in rats fed high-fructose diet or quinoa diet were within the normal range for total protein [29]. Albumin level in all rats was similar because diets which were used in our study were protein sufficient.

The similarity in levels of essential elements in rats fed control, high-fructose or quinoa diets might be due to the fact that all diets were supplemented with mix of minerals.

\section{Conclusions}

The ancient seeds investigated in the present study interact with most, but not all adverse effects of fructose on lipid profile, e.g. the doses of quinoa seeds used in our model did not inhibit an increase of triglycerides caused by fructose. In all groups affected by dietary fructose, the enhanced uric acid concentration proved metabolic disturbance, while decrease in alkaline phosphatase activity seems to be a new observation associated with influence of fructose on the rat organism. We proofed that quinoa exerts many advantageous biochemical effects and can serve as a promising component of functional food.

Acknowledgements This work was supported in part by the grants from Polish Ministry of Science and Higher Education, project PBZ-KBN 094/P06/2003/08 and K/ZDS/001294. The excellent technical assistance of Dr. M. Krośniak is highly appreciated.

Open Access This article is distributed under the terms of the Creative Commons Attribution Noncommercial License which permits any noncommercial use, distribution, and reproduction in any medium, provided the original author(s) and source are credited.

\section{References}

1. Tappy L, Le K, Tran Ch, Paquot N (2010) Fructose and metabolic diseases: New findings, new questions. Nutrition. doi:10.1016/j. nut.2010.02.014
2. Ackerman Z, Oron-Herman M, Grozovski M, Rosenthal T, Pappo O, Link G, Sela BA (2005) Fructose-induced fatty liver disease. Hepatic effects of blood pressure and plasma triglyceride reduction. Hypertension 45:1012-1018

3. Pasko P, Barton H, Zagrodzki P, Izewska A, Krosniak M, Gawlik M, Gawlik M, Gorinstein S (2010) Effect of diet supplemented with quinoa seeds on oxidative status in plasma and selected tissues of high fructose-fed rats. Plant Foods Hum Nutr 65:146-151

4. Busserolles J, Gueux E, Rock E, Mazur A, Rayssiguier Y (2002) Substituting honey for refined carbohydrates protects rats from hypertriglyceridemic and prooxidative effects of fructose. J Nutr 132:3379-3382

5. Gorinstein S, Vargas OJM, Jaramillo NO, Salas IA, Ayala ALM, Arancibia-Avila P, Toledo F, Katrich E, Trakhtenberg S (2007) The total polyphenols and the antioxidant potentials of some selected cereals and pseudocereals. Eur Food Res Technol 225:321-328

6. Paśko P, Sajewicz M, Gorinstein S, Zachwieja Z (2008) Analysis of selected phenolic acids and flavonoids in Amaranthus cruentus and Chenopodium quinoa seeds and sprouts by HPLC. Acta Chromomatogr 20:661-672

7. Ryan E, Galvin K, O'Connor T, Maguire A, O’Brien N (2007) Phytosterol, squalene, tocopherol content and fatty acid profile of selected seeds, grains, and legumes. Plant Foods Hum Nutr 62:85-91

8. Gonzalez J, Roldan A, Gallardo M, Escudero T, Prado F (1989) Quantitative determination of chemical compounds with nutritional value from Inca crops: Chenopodium quinoa (quinoa). Plant Foods Hum Nutr 39:331-337

9. Takao T, Watanabe N, Yuhara K, Itoh S, Suda S, Tsuruoka Y, Nakatsugawa K, Konishi Y (2005) Hypocholesterolemic effect of protein isolated from quinoa (Chenopodium quinoa Willd.) seeds. Food Sci Technol Res 11:161-167

10. Berger A, Gremaud G, Baumgartner M, Rein D, Monnard I, Kratky E, Geiger W, Burri J, Dionisi F, Allan M, Lambelet P (2003) Cholesterol - lowering properties of amaranth grain and oil in hamsters. Int J Vitam Nutr Res 73:39-47

11. Czerwiński J, Bartnikowska E, Leontowicz H, Lange E, Leontowicz M, Katrich E, Trakhtenberg S, Gorinstein S (2004) Oat (Avena sativa L.) and amaranth (Amaranthus hypochondriacus) meals positively affect plasma lipid profile in rats fed cholesterolcontaining diets. J Nutr Bioch 15:622-629

12. Escudero NL, Zirulnik F, Gomez NN, Mucciarelli SI, Gimenez MS (2006) Influence of a protein concentrate from Amaranthus cruentus seeds on lipid metabolism. Exp Biol Med 231:50-59

13. Kim HK, Kim MJ, Shin DH (2006) Improvement of lipid profile by amaranth (Amaranthus esculantus) supplementation in streptozoticin-induced diabetes rats. Ann Nutr Metab 50:277-281

14. Plate AY, Areas JA (2002) Cholesterol-lowering effect of extruded amaranth (Amaranthus cruenrus L.) in hypercholesterolemia rabbits. Food Chem 76:1-6

15. Tomotake H, Yamamoto N, Kitabayashi H, Kawakami A, Kayashita J, Ohinata H, Karasawa H, Kato N (2007) Preparation of tartary buckwheat protein product and its improving effect on cholesterol metabolism in rats and mice fed cholesterol-enriched diet. J Food Sci 72:528-533

16. Hellerstein MK (2002) Carbohydrate-induced hypertriglyceridemia: Modifying factors and implications for cardiovascular risk. Curr Opin Lipidol 13:33-40

17. Girard A, Madani S, Boukortt F, Cherkaoui-Malki M, Belleville J, Prost J (2006) Fructose-enriched diet modifies antioxidant status and lipid metabolism in spontaneously hypertensive rats. Nutrition 22:758-766

18. Wang M, Liu J, Gao J, Parry J, Wei Y (2009) Antioxidant activity of tartary buckwheat bran extract and its effect on the lipid profile of hyperlipidemic rats. J Agric Food Chem 57:51065112 
19. Lee S, Simons A, Murphy P, Hendrich S (2005) Group B soyasaponins lowered plasma cholesterol and increased fecal bile acids in female golden syrian hamsters. Exp Biol Med 230:472-478

20. Benado M, Alcantara C, De la Rosa R, Ambrose M, Mosier K, Kern M (2004) Effects of various levels of dietary fructose on blood lipids of rats. Nutr Res 24:565-571

21. Lewis GF, Murdoch S, Uffelman K, Naples M, Szeto L, Albers A, Adeli K, Brunzell JD (2004) Hepatic lipase mRNA, protein, and plasma enzyme activity is increased in the insulin-resistant, fructose-fed Syrian golden hamster and is partially normalized by the insulin sensitizer rosiglitazone. Diabetes 53:2893-2900

22. Busserolles J, Gueux E, Rock E, Demigne Ch, Mazur A, Rayssiguier Y (2003) Oligofructose protects against the hypertriglyceridemic and pro-oxidative effects of a high fructose diet in rats. J Nutr 133:1903-1908

23. Ardiansyah SH, Koseki T, Ohinata K, Hashizume K, Komai M (2006) Rice bran fraction improves blood pressure, lipid profile, and glucose metabolism in stroke-prone spontaneously hypertensive rats. J Agric Food Chem 54:1914-1920
24. Nakamura K, Yamagishi S, Matsui T, Inoue H (2005) Acarbose, an alpha-glucosidase inhibitor, improves insulin resistance in fructose-fed rats. Drugs Exp Clin Res 31:155-159

25. Thorburn AW, Crapo P, Beltz W, Wallace P, Wilztium J, Henry R (1989) Lipid metabolism in non-insulin-dependent-diabetes: Effects of long term treatment with fructose-supplemented mixed meals. Am J Clin Nutr 50:1015-1022

26. Anurag P, Anuradha CV (2002) Metformin improves lipid metabolism and attenuates lipid peroxidation in high fructose-fed rats. Diabetes Obes Metab 4:36-42

27. Yamamoto T, Moriwaki Y, Takahashi S, Tsutsumi Z, Yamakita JI, Higashino K (1999) Effects of fructose and xylitol on the urinary excretion of adenosine, uridine, and purine bases. Metabolism 48:520-524

28. Kizhner T, Werman MJ (2002) Long-term fructose intake: biochemical consequences and altered renal histology in the male rat. Metabolism 51:1538-1547

29. Obatolu V, Ketiku A, Adebowale E (2003) Effect of feeding maize/legume mixtures on biochemical indices in rats. Ann Nutr Metab 47:170-175 